\title{
Flow dynamics of a dandelion pappus: A linear stability approach
}

\author{
P. G. Ledda, ${ }^{1}$ L. Siconolfi, ${ }^{1}$ F. Viola,${ }^{2}$ S. Camarri, ${ }^{3}$ and F. Gallaire ${ }^{1}$ \\ ${ }^{1}$ Laboratory of Fluid Mechanics and Instabilities, École Polytechnique Fédérale de Lausanne, \\ CH-1015 Lausanne, Switzerland \\ ${ }^{2}$ Physics of Fluids Group and Max Planck Center Twente, MESA+ Institute and J. M. Burgers Centre for \\ Fluid Dynamics, Faculty of Science and Technology, University of Twente, P.O. Box 217, \\ NL-7500 AE Enschede, The Netherlands \\ ${ }^{3}$ Department of Industrial and Civil Engineering, Università di Pisa, Pisa, Italy
}

(Received 25 February 2019; published 2 July 2019)

\begin{abstract}
The study and control of flow instabilities is a key problem in aerodynamics. Aircrafts are designed not only to generate the lift force needed to balance their weight but, more importantly, to be stable and reasonably steady when in cruise conditions. Similar flow stability properties are naturally achieved by biological flying objects such as the dandelion seeds that are transported by the wind owing to a disklike structure called a pappus. The pappus creates a parachute flow configuration and is a remarkable prototype of how the wake, which would be unsteady if the pappus was completely impermeable, can be stabilized by changing the body structure so as to allow the flow to pass through. We approach the problem using the approximation of an anisotropic and nonhomogeneous rigid porous disk, combined with the linear stability analysis technique. The results show the presence of a mean porosity threshold beyond which the flow is always characterized by a separated, steady, and axisymmetric recirculating vortex ring. We compare our results with those of real dandelion pappi. The threshold is very close to the experimentally observed values of porosity, explaining why the morphology of the pappus promotes a steady wake regime.
\end{abstract}

DOI: 10.1103/PhysRevFluids.4.071901

Bifurcations are responsible for abrupt changes in the topological structure of fluid flows and linear stability analysis is a fundamental tool in order to deepen our understanding of the physical mechanism driving these transitions [1-4]. The characterization of the different flow regimes as a function of the bifurcation parameters delineates strategies to manipulate the flow so as to achieve desired stability properties. Considering the passive control of wakes past bluff bodies, the strategies often consist in slight changes of their shape or in modifications of the solid properties. This is the case when porous bodies are considered, instead of impermeable ones. Specifically, dealing with flows that can pass around and through porous objects is an effective strategy to obtain significant modifications in the wake patterns [5-10]. The work by Cummins et al. [11] offers a good example in this regard. In that paper, the authors studied the dispersal flight of the dandelion fruit, known to be promoted by the bristly pappus present at its apex. The long-distance dispersal of the dandelion seeds benefits from convective updrafts, which allow them to disperse over $100 \mathrm{~m}[12,13]$, or even more. In Ref. [11] the influence of the pappus porosity on its dynamics is studied using experimental measurements on real pappi in freely flying tests and on microfabricated silicon disks mimicking the pappus behavior. The authors showed that the organization of the filaments of the pappus corresponds to high values of porosity that is tuned in order to have a steady separated vortex ring in the wake, this being an essential feature for a stable and long dispersal flight.

In this Rapid Communication, we provide a theoretical explanation of the flow behavior documented in Ref. [11], so as to prove that the characteristics of the pappus allow us to produce a 
(a)

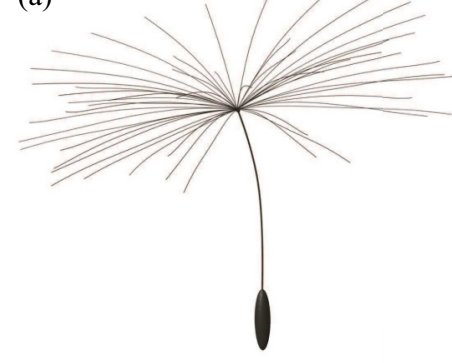

(b)

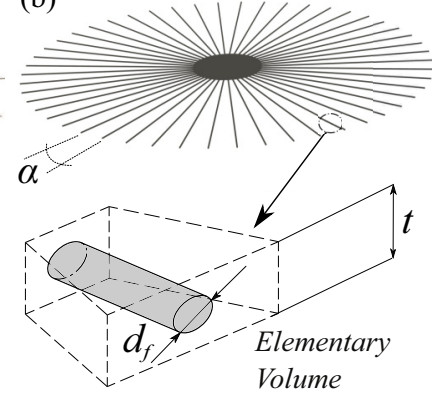

(c)

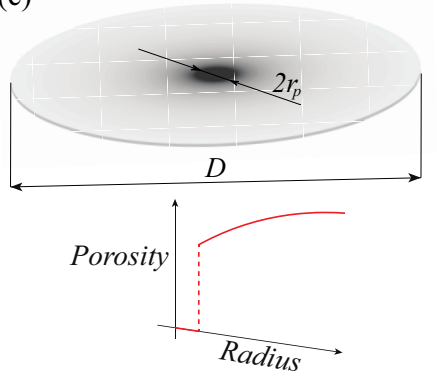

FIG. 1. (a) Sketch of a dandelion pappus. (b) Simplified discrete model of a pappus, characterized by the filament number $n_{f}$ and diameter $d_{f}$. (c) Continuous porous disk considered to model the flow past a pappus, where porosity and permeability are functions of the disk radius.

parachutelike configuration, where the generated wake promotes steady flow regimes. To this aim, we tackle the problem combining bifurcation analysis and averaging technique for nonhomogeneous porous media. The flow through porous media could, in principle, be studied using direct numerical simulations as done in Ref. [14], where the flow past a sphere with discretized porous elements on its surface is solved, or using a lattice Boltzmann method, as proposed by Ref. [15] to study the flow within a channel with a hairy surface. However, the long computational times owing to the bottleneck of the porous region discretization make these approaches inadequate for a parametric study, especially when a wide range of length scales is involved, as is the case here (with three orders of magnitude between the pappus and the filament diameter).

The first step of the present analysis consists in defining a simplified geometry of the pappus used to derive the homogenized and continuous characteristics of the corresponding porous disk, i.e., porosity and permeability. Considering an elementary volume in the porous medium, the porosity $\phi$ is defined as the ratio between the volume occupied by the fluid and the total volume, while the permeability tensor $\boldsymbol{K}$ is linked with the resistance that the fluid experiences in order to pass through. Following the characterization of the dandelion pappus reported in Ref. [11], we approximate a real pappus, sketched in Fig. 1(a) as a flat thin disk of diameter $D=14.8 \mathrm{~mm}$, made by two concentric regions [see Fig. 1(b)]: (i) an impervious central disk of radius $r_{p} \approx 2.5 \% D=370 \mu \mathrm{m}$, corresponding to the pulvinus of the pappus, the region where all the filaments are attached to the fruit, and (ii) an annular region composed by an array of $n_{f}$ circular cylinders of diameter $d_{f} \approx 0.11 \% D=16.2 \mu \mathrm{m}$. The thickness of the equivalent porous disk is here set to $t=2 d_{f}$ (as in the experiments of Ref. [16]) and its effect on the stability results is then studied in the final part of this Rapid Communication by varying it from $t=d_{f}$ to $t=5 d_{f}$. We define a cylindrical coordinate system $(x, r, \theta)$, the origin of which coincides with the center of the disk, and the $x$ direction is parallel to the inlet velocity. The porosity and permeability will result in a function of the disk radius $r$, as in the model sketched in Fig. 1(c). We introduce the local porosity $\phi(r)$ and the ratio between the voids area of the projected disk and its total area, here referred to as mean porosity $\Phi$,

$$
\phi(r)=1-\frac{n_{f} d_{f}^{2}}{8 t r}, \quad \Phi=1-\frac{n_{f} d_{f}\left(D / 2-r_{p}\right)+\pi r_{p}^{2}}{\pi(D / 2)^{2}}
$$

In the limit of quasiparallel circular fibers, and owing to the small angle between two filaments [ $\alpha \sim 10^{-2}$ rad, see Fig. 1(b)], the permeability tensor components can be written as follows $[17,18]$,

$$
K_{x x}=K_{\theta \theta}=\frac{\pi \phi(1-\sqrt{1-\phi})^{2} d_{f}^{2}}{96(1-\phi)^{3 / 2}}, \quad K_{r r}=\frac{(5.299-2.157 \phi) \phi^{2} d_{f}^{2}}{192(1-\phi)^{2}},
$$


(a)

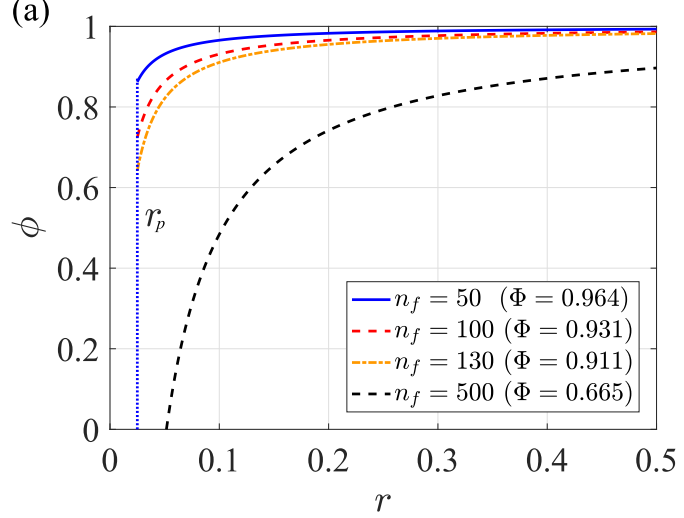

(b)

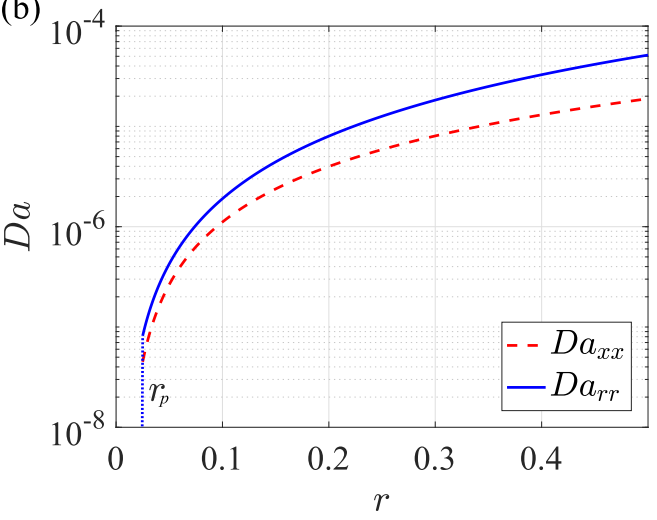

FIG. 2. (a) Typical porosity distribution for different numbers of filaments; (b) permeability variation along the radius when $n_{f}=100\left(D a_{\theta \theta}=D a_{x x}\right)$.

where $K_{x x}, K_{r r}$, and $K_{\theta \theta}$ denote the axial, the radial, and the azimuthal components of the permeability tensor of a parallel array of circular fibers, while all the cross components are zero.

In this study, we explore a wide range of mean porosities $\Phi$, here obtained keeping constant the diameter of the filaments $d_{f}$ and varying their number $n_{f}$. When a large number of filaments is considered, the radius of the pulvinus $r_{p}$ is increased so as to ensure $\phi\left(r=r_{p}\right)=0$. In Fig. 2(a), the porosity is reported as a function of the disk radius for different values of $n_{f}$. For the case $n_{f}=100$, the local porosity varies from $\phi=0.725$ to $\phi=0.986$, with a corresponding mean value $\Phi=0.931$. In Fig. 2(b) we report the permeability components as functions of the radius. According to Ref. [11], the dandelion pappus is composed by a number of filaments in the range $95 \leqslant n_{f} \leqslant$ 106.

We turn now to briefly describe the mathematical model considered in the global stability analysis carried out here. The motion outside of the porous disk is described by the unsteady incompressible Navier-Stokes equations that can be written in nondimensional form as

$$
\partial_{t} \boldsymbol{u}+\boldsymbol{u} \cdot \nabla \boldsymbol{u}+\nabla p-\frac{1}{\operatorname{Re}} \nabla^{2} \boldsymbol{u}=\mathbf{0}, \quad \nabla \cdot \boldsymbol{u}=\mathbf{0},
$$

where $\boldsymbol{u}$ is the velocity vector and $p$ the pressure field. The equations are normalized considering as reference quantities the uniform constant terminal velocity $U_{\infty}$ and the diameter of the disk $D$; the Reynolds number is defined as $\operatorname{Re}=U_{\infty} D / v$, where $v$ is the kinematic viscosity of the fluid. In the porous region, the motion of the fluid is described in average quantities considering a model based on the Brinkman formulation [19-24], where also the convective terms in the momentum equations are retained (see Ref. [10] for details),

$$
\frac{1}{\phi} \partial_{t} \boldsymbol{u}+\frac{1}{\phi^{2}} \boldsymbol{u} \cdot \nabla \boldsymbol{u}+\nabla p-\frac{1}{\phi \operatorname{Re}} \nabla^{2} \boldsymbol{u}+\frac{1}{\operatorname{Re}} \boldsymbol{D} \boldsymbol{a}^{-1} \boldsymbol{u}=\mathbf{0}, \quad \nabla \cdot \boldsymbol{u}=\mathbf{0} .
$$

The nondimensional permeability tensor $\boldsymbol{D a}$ is defined as $\boldsymbol{D a}=\boldsymbol{K} / D^{2}$.

Equations (3) and (4) are completed imposing Dirichlet boundary conditions on the inlet and on the lateral boundary, i.e., $u_{x}=1$ and $u_{r}=u_{\theta}=0$, stress-free conditions on the outflow boundary, and continuity of the velocity and pressure as interface conditions at the fluid-solid boundaries.

Following a linear stability approach, the flow is decomposed as $\boldsymbol{q}=\boldsymbol{Q}_{b}(x, r)+\varepsilon \boldsymbol{q}^{\prime}(x, r, \theta, t)$, where $\boldsymbol{Q}_{b}=\left(\boldsymbol{U}_{b}, p_{b}\right)$ is usually referred to as the base flow and $\boldsymbol{q}^{\prime}=\left(\boldsymbol{u}^{\prime}, p^{\prime}\right)$ is the unsteady perturbation with small amplitude $\varepsilon \ll 1$. Introducing this flow decomposition in Eqs. (3) and (4), we get at zero order the steady governing equations for the base flow, which correspond to the steady and axisymmetric version of the same equations to be completed with suitable regularity conditions at the axis $\left(U_{b r}=\frac{\partial U_{b x}}{\partial r}=0\right.$ for axisymmetric solutions). At order $\varepsilon^{1}$, we obtain the 


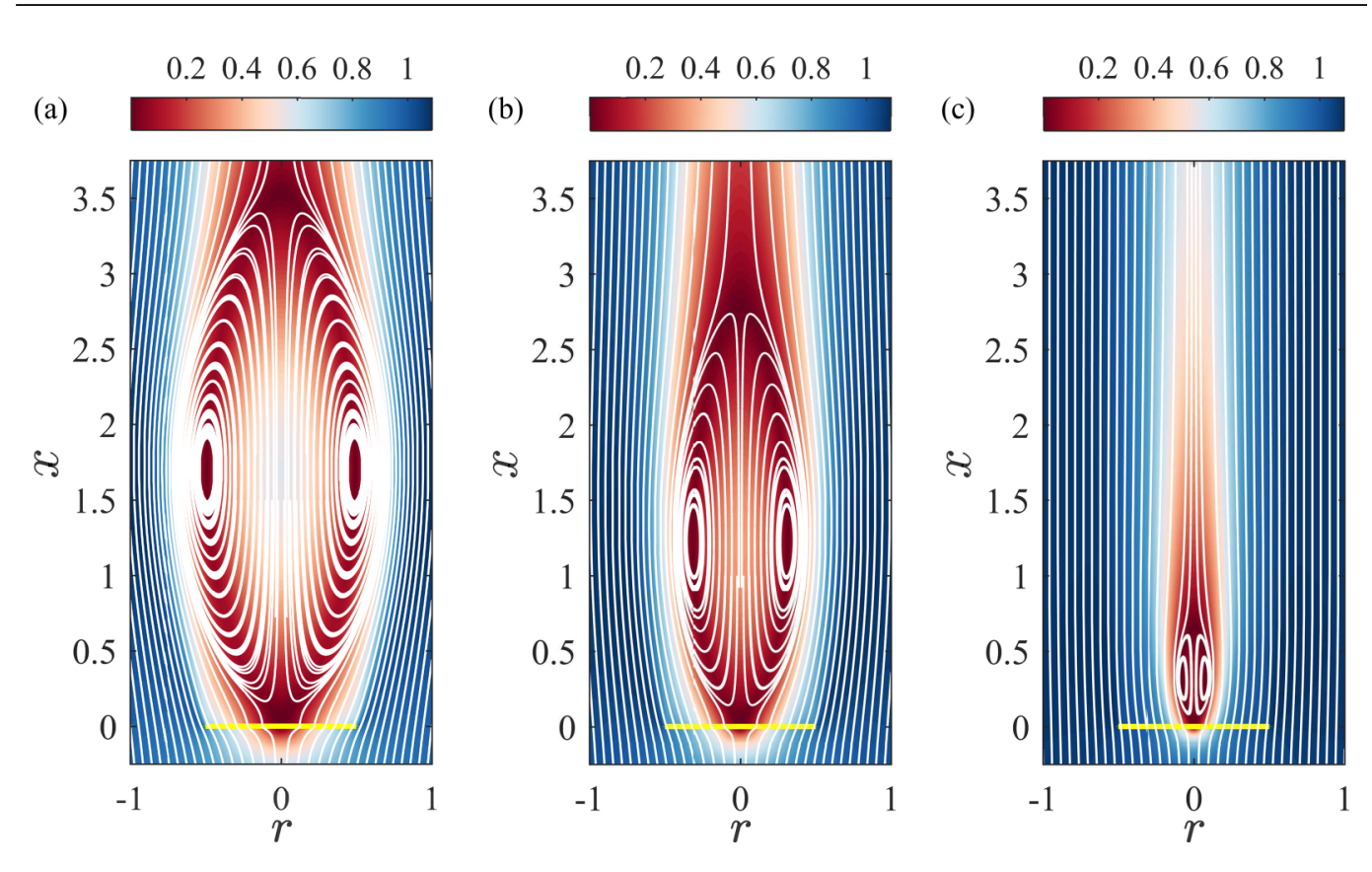

FIG. 3. Steady and axisymmetric solution of the flow equations for (a) $n_{f}=130$ ( $\left.\Phi=0.911\right)$, (b) $n_{f}=$ $100(\Phi=0.931)$, (c) $n_{f}=50(\Phi=0.964)$. The isocontours represent the velocity magnitude.

evolution of the unsteady perturbation that is expanded in Fourier modes in the azimuthal direction $\boldsymbol{q}^{\prime}(x, r, \theta, t)=\hat{\boldsymbol{q}}(x, r) \exp (\operatorname{im} \theta+\sigma t)$, where the integer $m$ is the azimuthal wave number and $\sigma$ is a complex number whose real and imaginary parts are the perturbation growth rate and frequency. The resulting eigenvalue problem reads [25]

$$
\sigma \hat{\boldsymbol{u}}+\boldsymbol{U}_{b} \cdot \nabla_{m} \hat{\boldsymbol{u}}+\hat{\boldsymbol{u}} \cdot \nabla_{0} \boldsymbol{U}_{b}+\nabla_{m} \hat{p}-\frac{1}{\operatorname{Re}} \nabla_{m}^{2} \hat{\boldsymbol{u}}=\mathbf{0}, \quad \nabla_{m} \cdot \hat{\boldsymbol{u}}=\mathbf{0}
$$

in the fluid region, and

$$
\frac{1}{\phi} \sigma \hat{\boldsymbol{u}}+\frac{1}{\phi^{2}}\left(\boldsymbol{U}_{b} \cdot \nabla_{m} \hat{\boldsymbol{u}}+\hat{\boldsymbol{u}} \cdot \nabla_{0} \boldsymbol{U}_{b}\right)+\nabla_{m} \hat{p}-\frac{1}{\phi \operatorname{Re}} \nabla_{m}^{2} \hat{\boldsymbol{u}}+\frac{1}{\operatorname{Re}} \boldsymbol{D} \boldsymbol{a}^{-1} \hat{\boldsymbol{u}}=\mathbf{0}, \quad \nabla_{m} \cdot \hat{\boldsymbol{u}}=\mathbf{0},
$$

in the porous domain, where the operators are defined as follows,

$$
\nabla_{m} p=\left[\begin{array}{c}
\frac{\partial p}{\partial x} \\
\frac{\partial p}{\partial r} \\
\frac{i m p}{r}
\end{array}\right], \quad \nabla_{m} \boldsymbol{u}=\left[\begin{array}{ccc}
\frac{\partial u_{x}}{\partial x} & \frac{\partial u_{x}}{\partial r} & \frac{i m}{r} u_{x} \\
\frac{\partial u_{r}}{\partial x} & \frac{\partial u_{r}}{\partial r} & \frac{i m}{r} u_{r}-\frac{u_{\theta}}{r} \\
\frac{\partial u_{\theta}}{\partial x} & \frac{\partial u_{\theta}}{\partial r} & \frac{i m}{r} u_{\theta}+\frac{u_{r}}{r}
\end{array}\right], \quad \nabla_{m} \cdot \boldsymbol{u}=\frac{\partial u_{x}}{\partial x}+\frac{1}{r} \frac{\partial\left(r u_{r}\right)}{\partial r}+\frac{i m}{r} u_{\theta},
$$

and $\nabla_{m}^{2} \boldsymbol{u}=\nabla_{m} \cdot\left(\nabla_{m} \boldsymbol{u}\right)$. We focus on the displacement mode $m=1$, that is the least stable in the case of solid axisymmetric bodies [25], which requires the regularity conditions $\frac{\partial \hat{u}_{r}}{\partial r}=\hat{u}_{x}=\frac{\partial \hat{u}_{\theta}}{\partial r}=0$ at the axis. For the numerical implementation, we use the open source software FREEFEM $++[26]$, as described in Ref. [10], to which we refer also for the convergence study. Typically, 200000 degrees of freedom are used and the boundaries are located at $r_{\infty}=20, x_{-\infty}=-25, x_{+\infty}=50$. The pulvinus region is treated as a solid boundary, on which the impermeability condition is imposed using a homogeneous Dirichlet boundary condition (i.e., $\boldsymbol{u}=\mathbf{0}$ ) on its border.

We turn now to present the results of our analysis. In Fig. 3, three steady flow solutions $\boldsymbol{Q}_{b}$ are shown for different numbers of the filaments $n_{f}$, namely, $n_{f}=130,100,50$ ( $\Phi=$ $0.911,0.931,0.964)$, at fixed Reynolds number $\mathrm{Re}=400$, which can be considered a typical value for the dispersal flight of dandelion seeds [11]. We recall that $\mathrm{Re}$ is based on the disk 

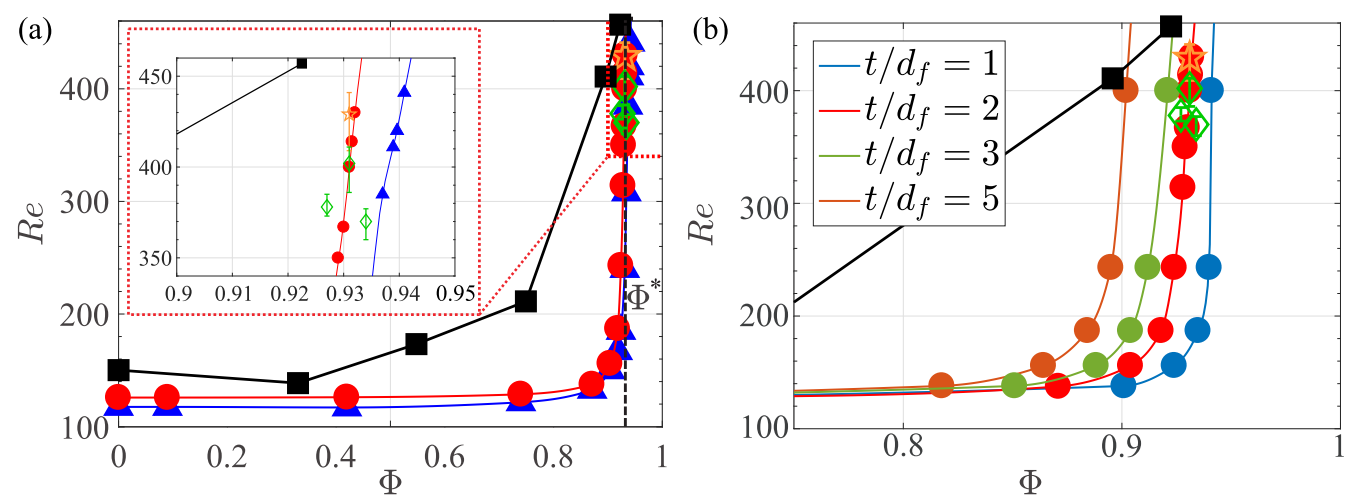

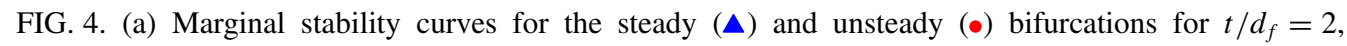
with a comparison with the results of Ref. [11] for porous disks (๘), freely falling dandelion pappi for $n_{f}=95,100,106(\diamond)$, and the critical Reynolds number for the dandelion pappus $(\downarrow)$. The vertical line depicts the threshold of mean porosity for unconditional stability $\Phi^{*}$ and the inset zooms in the threshold region. (b) Effect of the disk thickness on the marginal stability curve of the unsteady bifurcation.

diameter, which yields a local Reynolds number based on the filament diameter in the order of $\operatorname{Re}_{f}=\left(d_{f} / D\right) \operatorname{Re} \sim 10^{-1}$, which implies that the flow around the filament does not separate and the Stokes regime prevails. The flow patterns in the near wake consist of vortex rings that are detached from the disk base for a large extent. The permeability of the disks increases when the number of the filaments $n_{f}$ decreases, with a consequent reduction in the size of the recirculation regions both in the $x$ and in $r$ directions [Figs. 3(a)-3(c)]. At least for the cases shown here, the vortex rings remain partially attached to the body in the impermeable region that corresponds to the pulvinus of the dandelion, at difference with the case of a homogeneous porous disk [9], in which the recirculation regions tend instead to move away from the body when higher permeabilities are considered. We present now the results of the stability analysis. As the value of Re is increased, the wake of a solid disk of infinitesimal thickness shows two successive helical bifurcations; the first is steady while the second is periodic in time [25,27]. The same type of bifurcation scenario is found here, but with different critical Reynolds numbers $\operatorname{Re}_{\mathrm{cr}}$ that strongly depend on the permeability.

Referring to the neutral curves for the two bifurcations reported in solid blue and red lines in Fig. 4(a), the $\mathrm{Re}_{\mathrm{cr}}$ for the onset of the instabilities are constant and equal to those of the impermeable zero-thickness case $\left(\mathrm{Re}_{\mathrm{cr}}^{\mathrm{I}}=117\right.$ and $\left.\mathrm{Re}_{\mathrm{cr}}^{\mathrm{II}}=125\right)$ for mean porosities in the range $0 \leqslant \Phi \leqslant 0.7$. Starting from $\Phi \simeq 0.7$, the value of $\operatorname{Re}_{\text {cr }}$ increases with $\Phi$, up to a value of $\Phi^{*} \simeq 0.93$, where the two neutral stability curves diverge. Thus, steady and axisymmetric base flows are linearly stable for $\Phi>0.93$ that corresponds, according to our porous model, to $n_{f} \simeq 100$, which is close to that typically observed in a dandelion pappus. Furthermore, Fig. 4(b) shows the effect of the disk thickness on the marginal stability curve of the unsteady bifurcation by varying the thickness from $t / d_{f}=1$ to $t / d_{f}=5$. It is found that, as the thickness increases, the disk wake is stabilized and the critical value of the mean porosity $\Phi_{\mathrm{cr}}$ decreases. Nevertheless, $\Phi_{\mathrm{cr}}$ is seen to decrease only weakly with $t$, since only a deviation $\Delta \Phi_{\mathrm{cr}}=0.04$ occurs when the disk thickness is varied from $t=d_{f}$ to $t=5 d_{f}$, which is in line with the experimental results of Ref. [11] ( $\Delta \Phi_{\text {expt }}=0.02$ including the uncertainties due to the the experimental measurements). These results are also in line with what was observed for the flow past a two-dimensional rectangular cylinder with an isotropic and homogenous porosity [10]. Please note that, although the first bifurcation breaks the axisymmetry, yielding a nonaxisymmetric three-dimensional (3D) steady state, it is relevant to investigate the second bifurcation of the axisymmetric base flow since the three-dimensional dynamics of the flow around a solid disk can be explained as a nonlinear interaction between the unstable modes of the axisymmetric base flow $[27,28]$. In these terms, the analysis of the successive bifurcations of the 
(a)

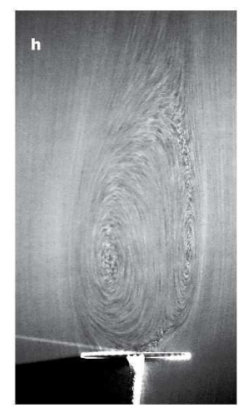

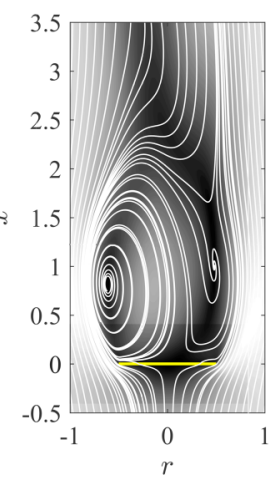

(b)

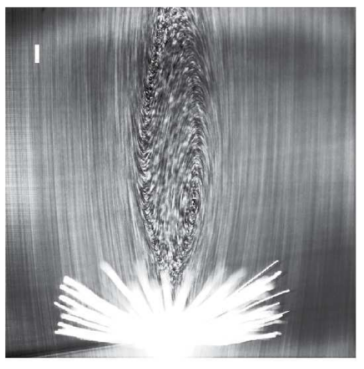

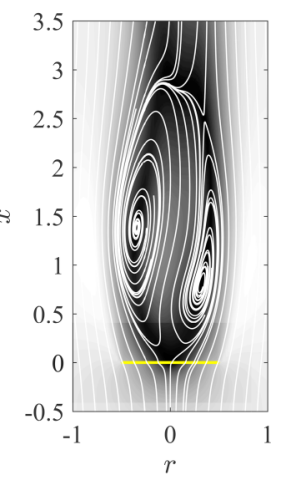

FIG. 5. For both panels: Instantaneous experimental visualization reproduced from Ref. [11] (on the left), and reconstruction of the flow using a linear combination of the two modes (on the right), at the marginal stability of the unsteady mode $(\operatorname{Re} \simeq 129$ for $\Phi=0.75, \operatorname{Re} \simeq 400$ for $\Phi=0.931)$. (a) $\Phi=0.75$, (b) $\Phi=$ 0.931 (case equivalent to the dandelion pappus).

steady and axisymmetric flow past a porous disk is meaningful to assess the overall stability and steadiness of the pappus wake. Indeed, the neutral curves predicted by the stability analysis shown in Fig. 4(a) are in good agreement with the experimental data for freely falling tests (in which the wake is steady) of real pappi, as for their critical Reynolds number for the onset of the unsteady wake [11]. This result suggests that the morphology of the pappus seems to be indeed tuned in order to ensure a stable and steady flow regime.

For the sake of completeness, we also report in Fig. 4(a) the results from Ref. [11] concerning experiments performed on equivalent porous disks mimicking the dandelion pappus. Even though the qualitative behavior looks similar, we can notice discrepancies between this set of experimental data and our numerical results. In our opinion, these differences are mainly due to the following aspects: (i) First, in the experiments, the microfabricated disks were similar to Fig. 1(b), but with filaments of rectangular instead of circular cross sections; (ii) second, variations of porosity are obtained in the experimental work changing the size of the filaments and keeping constant their number at $n_{f}=42$. These two aspects lead to a strong modification of the spatial distribution of the permeability and porosity [see Eqs. (1) and (2)], with a consequent modification of the $\mathrm{Re}_{\mathrm{cr}}$ for the onset of both bifurcations.

As the final step, we present a qualitative comparison between the flow patterns observed in the experiments documented in Ref. [11] and the spatial distribution of the resulting flow through the successive bifurcations here identified via a stability analysis. The unsteady wake is characterized by a spiraling mode, with a time-periodic shedding of large-scale vortical structures. The numerical flow fields reported in Fig. 5 have been obtained using a suitable linear combination between the base flow and the linear modes, at the marginal stability of the unsteady mode.

This work provides rigorous mathematical support in the framework of bifurcation theory to the modeling of the dandelion pappus as a porous disk, originally proposed in the experiments of Cummins et al. [11]. This stability analysis, which accounts for the space variability of porosity and permeability, confirms that the morphology of the pappus is able to promote stable and steady wake flow regimes that allow the long-distance dispersal of the dandelion seeds. Although the geometry and nondimensional numbers are set to match the ones of a real pappus, the model only embeds some representative features of what occurs in nature and it could be refined in several ways. For instance, in the current study the permeability properties of the disk are steady and axisymmetric, thus neglecting the elastic deformation and the three-dimensional dynamics of the filaments due to the fluctuating hydrodynamics loads. Nevertheless, the linear stability modes are seen to qualitatively well reproduce the unsteady flow structures observed experimentally in a supercritical condition. We conclude by noting that we provide here an example of how we 
can make use of linear stability models on simplified flow configurations to theoretically explain observations derived from experiments. In particular, linear approaches are particularly suitable when low Reynolds number environments are considered, making the biological systems the perfect candidates for these kinds of studies.

F.V. acknowledges the support of the Swiss National Science Foundation (Grant No. P2ELP2 172320). P.G.L. and F.G. acknowledge the financial support of the Swiss National Science Foundation (Grant No. 200021_178971).

[1] P. A. Monkewitz, The absolute and convective nature of instability in two-dimensional wakes at low Reynolds numbers, Phys. Fluids 31, 999 (1988).

[2] P. Huerre and P. A. Monkewitz, Local and global instabilities in spatially developing flows, Annu. Rev. Fluid Mech. 22, 473 (1990).

[3] F. Giannetti and P. Luchini, Structural sensitivity of the first instability of the cylinder wake, J. Fluid Mech. 581, 167 (2007).

[4] F. Gallaire and P.-T. Brun, Fluid dynamic instabilities: Theory and application to pattern forming in complex media, Philos. Trans. R. Soc., A 375, (2017).

[5] I. P. Castro, Wake characteristics of two-dimensional perforated plates normal to an air-stream, J. Fluid Mech. 46, 599 (1971).

[6] T.-C. Jue, Numerical analysis of vortex shedding behind a porous square cylinder, Int. J. Numer. Methods Heat Fluid Flow 14, 649 (2004).

[7] X. Chen, P. Yu, S. H. Winoto, and H.-T. Low, Numerical analysis for the flow past a porous square cylinder based on the stress-jump interfacial-conditions, Int. J. Numer. Methods Heat Fluid Flow 18, 635 (2008).

[8] L. Zong and H. Nepf, Vortex development behind a finite porous obstruction in a channel, J. Fluid Mech. 691, 368 (2012).

[9] C. Cummins, I. M. Viola, E. Mastropaolo, and N. Nakayama, The effect of permeability on the flow past permeable disks at low Reynolds numbers, Phys. Fluids 29, 097103 (2017).

[10] P. G. Ledda, L. Siconolfi, F. Viola, F. Gallaire, and S. Camarri, Suppression of von Kármán vortex streets past porous rectangular cylinders, Phys. Rev. Fluids 3, 103901 (2018).

[11] C. Cummins, M. Seale, A. Macente, D. Certini, E. Mastropaolo, I. M. Viola, and N. Nakayama, A separated vortex ring underlies the flight of the dandelion, Nature (London) 562, 414 (2018).

[12] O. Tackenberg, P. Poschlod, and S. Kahmen, Dandelion seed dispersal: The horizontal wind speed does not matter for long-distance dispersal - it is updraft! Plant Biol. 5, 451 (2003).

[13] R. Nathan, F. M. Schurr, O. Spiegel, O. Steinitz, A. Trakhtenbrot, and A. Tsoar, Mechanisms of longdistance seed dispersal, Trends Ecol. Evol. 23, 638 (2008).

[14] G. A. Zampogna, J. Magnaudet, and A. Bottaro, Generalized slip condition over rough surfaces, J. Fluid Mech. 858, 407 (2019).

[15] J. Sundin and S. Bagheri, Interaction between hairy surfaces and turbulence for different surface time scales, J. Fluid Mech. 861, 556 (2019).

[16] Q. Meng, Q. Wang, K. Zhao, P. Wang, P. Liu, H. Liu, and L. Jiang, Hydroactuated configuration alteration of fibrous dandelion pappi: Toward self-controllable transport behavior, Adv. Funct. Mater. 26, 7378.

[17] J. Van der Westhuizen and J. P. Du Plessis, An attempt to quantify fibre bed permeability utilizing the phase average Navier Stokes equation, Composites, Part A 27, 263 (1996).

[18] G. A. Zampogna and A. Bottaro, Fluid flow over and through a regular bundle of rigid fibres, J. Fluid Mech. 792, 5 (2016).

[19] H. C. Brinkman, A calculation of the viscous force exerted by a flowing fluid on a dense swarm of particles, Flow, Turbul. Combust. 1, 27 (1949).

[20] G. S. Beavers and D. D. Joseph, Boundary conditions at a natural permeable wall, J. Fluid Mech. 30, 197 (1967). 
[21] S. Whitaker, Flow in porous media I: A theoretical derivation of Darcy's law, Transp. Porous Media 1, 3 (1986).

[22] S. Whitaker, The Forchheimer equation: A theoretical development, Transp. Porous Media 25, 27 (1996).

[23] J. A. Ochoa-Tapia and S. Whitaker, Momentum transfer at the boundary between a porous medium and a homogeneous fluid-I. Theoretical development, Int. J. Heat Mass Transfer 38, 2635 (1995).

[24] W. P. Breugem, The influence of wall permeability on laminar and turbulent flows, Theory and simulations, Doctoral dissertation, TU Delft, Delft University of Technology, 2004.

[25] P. Meliga, J.-M. Chomaz, and D. Sipp, Unsteadiness in the wake of disks and spheres: Instability, receptivity and control using direct and adjoint global stability analyses, J. Fluids Struct. 25, 601 (2009).

[26] F. Hecht, New development in FREEFEM++, J. Numer. Math. 20, 251 (2012).

[27] D. Fabre, F. Auguste, and J. Magnaudet, Bifurcations and symmetry breaking in the wake of axisymmetric bodies, Phys. Fluids 20, 051702 (2008).

[28] P. Meliga, J.-M. Chomaz, and D. Sipp, Global mode interaction and pattern selection in the wake of a disk: A weakly nonlinear expansion, J. Fluid Mech. 633, 159 (2009). 